\title{
Association between Coronary Artery Disease and Left Ventricle Remodeling Parameters in Hypertensive Patients: A Cross-Sectional Study in a Limited Resource Setting
}

\author{
Badai Tiksnadi ${ }^{1}$ (i), Erwan Martanto ${ }^{1}$ (D) Abednego Panggabean ${ }^{1}$ (D) Ary Indriana Savitri ${ }^{2}$ (D) Alberta Claudia Undarsa ${ }^{1}$ (D) \\ ${ }^{1}$ Department of Cardiology and Vascular Medicine, Faculty of Medicine, Universitas Padjadjaran, Hasan Sadikin General \\ Hospital, Bandung, Indonesia; ${ }^{2}$ Department of Clinical Epidemiology, Julius Center for Health Science and Primary Care, \\ University Medical Center, Utrecht, The Netherlands
}

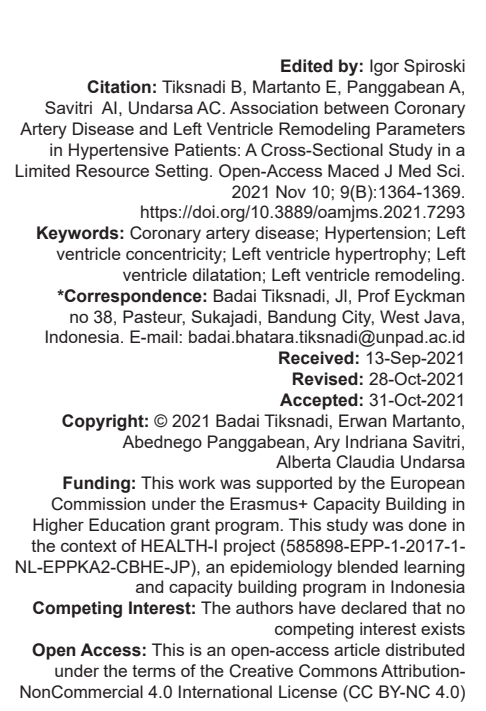

\begin{abstract}
BACKGROUND: Coronary artery disease (CAD) and hypertension are related with left ventricle (LV) remodeling however evidence about association between CAD and remodeling in hypertensive patient is still limited, especially in limited resource setting like Indonesia.

AIM: Evaluating impact of CAD on LV remodeling within hypertensive patients at tertiary referral hospital, Hasan Sadikin General Hospital Bandung, Indonesia.

METHOD: Cross-sectional study involving 120 hypertensive patients who visited cardiology outpatient clinic from September-December 2019 and underwent transthoracic echocardiography examination for any medical indications. LV remodeling parameters, such as mass (LV Mass Index [LVMi]), volume (end-diastolic volume/body surface area $[B S A]$ ), and relative wall thickness (RWT), were compared between CAD and non-CAD groups.

RESULTS: There were 108 patients to be analyzed, 12 patients were excluded due to technical difficulty $(n=9)$ and non-cooperative during interview $(n=3)$. Mean (standard deviation) age of patients was $56.9( \pm 11.8)$ years, $50(46.3 \%)$ patients were male, and median (interquartile range) hypertension duration was $3( \pm 4.40)$ years. CAD was found in $40(37.0 \%)$ patients. In the adjusted analysis, patients with CAD had average $27.75 \mathrm{~g} / \mathrm{m}^{2}$ higher LVM $(95 \%$ confined interval $[\mathrm{Cl}] 2.03 ; 53.47 ; p=0.035)$ and $16.20 \mathrm{ml} / \mathrm{m}^{2}$ higher LV end-diastolic volume/BSA $(95 \%$ $\mathrm{Cl} 4.14 ; 28.25 ; p=0.009$ ) compared to those without. This was independent of age, duration of hypertension, consumption of antihypertensive therapy, and type-2 diabetes mellitus, but disappeared after heart failure (HF) was included in the study. CAD and non-CAD groups were not different, respectively, to RWT.

CONCLUSION: In hypertensive patients, CAD was independently associated with higher LV mass and volume.
\end{abstract} These associations, however, were largely explained by the presence of HF. CAD did not associate with RWT.

\section{Introduction}

Left ventricular (LV) remodeling refers to the changes of ventricular configuration, characterized by increment of LV mass, LV volume and diameter, and eventually geometry. 1 LV mass and volume as cardiac remodeling parameters particularly received greater focus as they are clinically relevant for determining prognosis and therapy [1], [2]. This morphology changes certainly involve molecular, cellular and interstitial changes of LV [3]. Echocardiography has been proven to be a reliable and precise measure of LV mass, thickness and configuration, aside from ventriculography, and nuclear magnetic resonance [1], [3], [4].

LV configuration transformation occurs in response to a variety of causes of myocardial injury and increasing wall stress, such as hypertension or myocardial infarction (MI) [1]. Changes in LV geometry due to hypertension were initially thought to be an adaptive process to compensate stress load, which involves activation of neurohormonal system [5], [6]. In chronic term, this initially adaptive response might become maladaptive. In maladaptive LV hypertrophy, those features are accompanied by the thinning of LV wall due to extracellular matrix degradation which consequently increases LV chamber volume, thus causing dilatation. Both, adaptive and maladaptive hypertrophies are characterized by increased heart size and weight [7].

In general, coronary artery disease (CAD) is also known for its relationship with LV remodeling. Remodeling of the coronary arteries involved atherosclerosis development which starts from endothelium disturbance, progressive formation of atherosclerotic plaque, and direct injury of MI. As ischemia and infarction develop, it will consecutively causing myocardial remodeling [8]. Hypertensive state 
itself is also associated with CAD, as the activation of neuro-humoral mechanisms such as renin-angiotensinaldosterone system in hypertension not only trigger the progressive increase in LV mass, but also further promote the progression of atherosclerosis. It is also known that hypertension increases the level of oxidative stress, one of the most important contributors of atherosclerosis, by altering normal endothelial function and supporting pro-inflammatory, prothrombotic, proliferative, and vasoconstrictor effects [9].

Until present, there were limited number of studies which focused on LV remodeling parameters in hypertensive patients in Indonesia, and none of them evaluated the impact of CAD. As with previous study, racial differences were also associated with cardiac structure and function among whom had longterm consequences of cardiac effects due to arterial afterload [10]. Moreover, previous LIFE echo sub study evaluate relation between clinical evidence of CAD and LV remodeling only in specific population (excluded aortic stenosis, symptomatic heart failure [HF], and/ or LV ejection fraction [LVEF] $<40 \%$ ). Whereas, this study took place in real clinical setting where patients commonly present with comorbidities. This study aimed to evaluate if the presence of CAD was associated with various $L V$ remodeling parameters (LV mass index [LVMi], relative wall thickness [RWT], LV enddiastolic volume [LVEDV]/body surface area [BSA], and concentricity) in hypertensive patients who underwent transthoracic echocardiography examination in Hasan Sadikin General Hospital in Bandung, Indonesia. The site represented the health-care facilities in a low-middle income country that has suboptimal hypertensive patients' evaluation.

\section{Materials and Methods}

\section{Study population}

This study was an observational cross-sectional study, conducted from September to December 2019. Subjects were all patients who visited Cardiology Outpatient Clinic in Hasan Sadikin General Hospital, who had hypertension and underwent transthoracic echocardiography examination for any medical indication. Subjects who provided informed consent and aged 18 years or older were recruited in the study. Exclusion criteria were echocardiography showing poor echo window (technical difficulties) and non-cooperative patients during interview. Hypertension status was based on patients' self-reported or patient's current consumption of antihypertensive medication(s), or direct blood pressure (BP) measurements at recruitment. BP assessments were done using the standard method of BP measurement using a digital tension measurement device $(A B N-100)$. BP measurements were taken after the subjects rested in a seated position quietly for at least $10 \mathrm{~min}$. Three $\mathrm{BP}$ measurements with a time interval of 1-2 $\min (\mathrm{s})$ between each measurement were recorded. If there was a consistent difference between arms of $>10 \mathrm{~mm} \mathrm{Hg}$ in repeated measurements, measurement from the arm with higher BP was used. $\mathrm{BP}$ was recorded as the average of the last two BP readings. Hypertension was defined as systolic BP $\geq 140 \mathrm{mmHg}$ or diastolic BP $\geq 90 \mathrm{mmHg}$. CAD status was based on clinician diagnosis (as stated in medical record) or patients' self-reported history of angina pectoris or prior Ml. Patients, who admitted to have hypertension, were further interviewed whether they had consumed antihypertensive medications routinely and were asked to recall the duration of hypertension. HF was defined by LVEF $<40 \%$. Type 2 diabetes mellitus (T2DM) and chronic kidney disease (CKD) were defined based on medical record. Valvular heart disease was defined according to echocardiography data and was classified into moderate or severe mitral stenosis, mitral regurgitation, aortic stenosis, or aortic regurgitation.

\section{Echocardiography}

Echocardiography was conducted in all patients at Cardiac Center of Hasan Sadikin General Hospital Bandung, West Java, Indonesia. It was performed according to the international guidelines from the European Association of Cardiovascular Imaging and the American Society of Echocardiography to ensure the accuracy of the results [11]. All echocardiography readings were confirmed by two consultants to reduce operator dependent bias. Echocardiography measurements were calculated using LVEF according to the modified Simpson rule; LV mass, LV volume, and LV wall thickness to demonstrate cardiac function. RWT was calculated using the following formula $(2 \times$ diastolic posterior wall thickness)/diastolic LV internal diameter. LV volume assessed based on LVEDV by BSA.

\section{Statistical analysis}

Analyses were performed using SPSS version 24.0 (SPSS Inc., Chicago, IL). Descriptive statistics were done to describe the baseline characteristics of both CAD and non-CAD groups. They were valued by means and standard deviation, medians and interquartile ranges (IQR), and also numbers and percentages. Univariate analysis for comparison of CAD and non-CAD groups was done using independent $t$ test, Mann-Whitney, and Chisquare, as appropriate. Multivariable analysis with linear regression test was done to adjust for several potential confounders. Age, hypertension duration, use of antihypertensive medication, and T2DM were a priori considered as potential confounders. We also run an explanatory analysis where HF (yes/no) was also included in the model. 


\section{Results}

\section{Prevalence of CAD and baseline characteristics of patients}

Of 120 patients who had hypertension, 108 of them were analyzed after excluding patients with poor echo window $(n=9)$ and non-cooperative to be interviewed $(n=3)$. The mean age of the patients was $56.94 \pm 11.8$ years, $46.3 \%(n=50)$ were male, and the median (IQR) hypertension duration was $3(4.4)$ years. CAD was found in 40 patients $(37.0 \%)$, DM in 15 patients (13.9\%), HF in 15 patients (13.8\%), CKD in eight patients $(7.4 \%)$, and valvular heart disease in seven patients $(6.5 \%)$. Among all comorbidities, only T2DM was found significantly higher in CAD patients $(22.5 \%$ vs. $8.8 \% ; p=0.047)$. Patients with CAD were significantly older $(p<0.001)$ and known to have had hypertension for longer duration than its counterparts $(p=0.002)$. At recruitment, only $59.3 \%$ of the patients were consuming antihypertensive medication routinely, with those with CAD had significantly higher proportion than those without $(92.5 \%$ vs. $39.7 \% ; p<0.001)$ Table 1.

Table 1: Baseline characteristics of CAD and non-CAD group in hypertensive patients

\begin{tabular}{|c|c|c|c|c|}
\hline Characteristics & All subjects & CAD $(n=40)$ & No CAD $(n=68)$ & $p$ \\
\hline Gender, male, n (\%) & $50(46.3)$ & $21(52.5)$ & $29(42.6)$ & $0.32^{\mathrm{a}}$ \\
\hline Age, years, mean (SD) & $56.94(11.82)$ & $62.38(8.05)$ & $53.75(12.55)$ & $<0.001^{t}$ \\
\hline $\mathrm{BMI}, \mathrm{kg} / \mathrm{m}^{2}$, mean $(\mathrm{SD})$ & $24.60(4.00)$ & $25.18(3.95)$ & $24.26(4.03)$ & $0.251^{b}$ \\
\hline $\begin{array}{l}\text { Known hypertension } \\
\text { duration, median (IQR) years }\end{array}$ & $3(4.40)$ & $4(0-30)$ & $1.5(0-40)$ & $0.002^{\mathrm{C}}$ \\
\hline $\begin{array}{l}\text { Using antihypertensive } \\
\text { medication routinely, } \mathrm{n}(\%)\end{array}$ & $64(59.3)$ & $37(92.5)$ & $27(39.7)$ & $<0.001^{\mathrm{a}}$ \\
\hline T2DM & $15(13.9)$ & $9(22.5)$ & $6(8.8)$ & 0.047 \\
\hline CKD & $8(7.4)$ & $4(10)$ & $4(5.9)$ & 0.122 \\
\hline VHD & $7(6.5)$ & $4(10)$ & $3(4.4)$ & 0.255 \\
\hline $\mathrm{HF}$ & $15(13.9)$ & $11(27.5)$ & $4(5.9)$ & $0.001^{\mathrm{a}}$ \\
\hline
\end{tabular}

\section{$C A D$ and cardiac remodeling in hypertensive patients}

Among hypertensive patients, CAD was associated with higher LVMi and LVEDV/BSA. Hypertensive patients with CAD had on average $39.15 \mathrm{~g} / \mathrm{m}^{2}$ higher LVMi (95\% confined interval [Cl] 18.00; 60.33, $\mathrm{p}<0.001$ ) and $27.75 \mathrm{~g} / \mathrm{m}^{2}$ higher LVMi
$(95 \% \mathrm{Cl} 2.03 ; 53.47, \mathrm{p}=0.035)$ in the crude and adjusted analysis, respectively. LVEDV/BSA were also higher in the CAD group as compared to the non-CAD group with mean difference of $12.36 \mathrm{ml} / \mathrm{m}^{2}(0.85 ; 23.86$, $\mathrm{p}=0.036)$ and $16.20 \mathrm{ml} / \mathrm{m}^{2}$ (4.14; 28.25, $\left.\mathrm{p}=0.009\right)$ in the crude and adjusted analysis, respectively. In the explanatory model where HF was included, these associations diminished. CAD was not associated with RWT (Table 2).

\section{Discussion}

We found that CAD associated with alteration in cardiac remodeling pattern of hypertensive patients. Hypertensive patients with CAD had significantly higher LV mass and larger LV volume than non-CAD group. Although both groups had preserved systolic function, CAD groups showed lower LVEF than non-CAD group. This findings were in line with previous similar study by Zabalgoitia et al., which was an echo sub-study done in a subset of 963 patients with stage I-III hypertension, which constitutes $11 \%$ of the entire LIFE Study subjects. Among the 963 patients, the CAD group also had larger LV volume and greater mass than the non-CAD group [12].

Association between $\mathrm{LVH}$ and CAD was reported by Kishi et al. It was reported that only among patients with the previous MI, LVMi was independently associated with myocardial ischemia as detected by single-photon emission CT. Various factors which might influence the increase in myocyte mass are increased myocardial oxygen demand, increased coronary vascular and minimal vascular resistance, decreased myocardial oxygen supply which depends on coronary blood flow, myocardial blood flow, and coronary flow reserve. As seen in hypertensive cardiomyopathy, LVM also contributes to increased myocardial fibrosis [13]. Apparently not only large $\mathrm{MI}$ has linkage to $\mathrm{LVH}$, but also subclinical atherosclerosis as assessed by coronary artery calcium may progress to hypertrophied LV [14]. Bidirectional causation relationship of $\mathrm{LVH}$ and CAD was seen, as in serial cardiac catheterization study,

Table 2: Association between CAD and remodeling parameters (LVMi, RWT, and LVEDV/BSA) in hypertensive patients

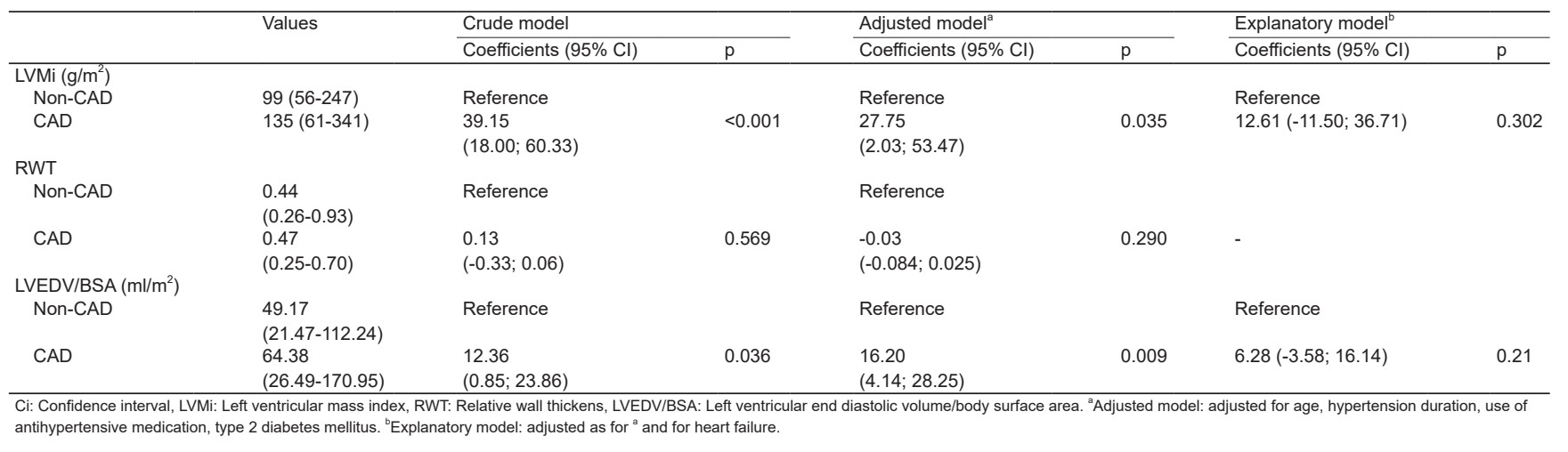


plaque rupture was more common among patient with LVH [14], [15]. As renin-angiotensin-aldosterone system was associated in both LVH and CAD pathogenesis, angiotensin II may be the common mediators which explain the linkage between them [16].

Dilated LV association with CAD might be driven on a histologic level, by myocyte hypertrophy and apoptosis as well as the increment of interstitial collagen as a response to myocardial injury. Post MI phase of LV remodeling started with fibrotic repair and scar formation, elongation, and thinning of the infarcted area. Meanwhile, hypertrophic myocyte elongation also occurs in non-infarcted zone resulting in increased mass, chamber dilation, and shifting to elliptical configuration [1].

Among all comorbidities, only T2DM was seen to be significantly different between CAD and non-CAD group. This result was predictable as we know CAD is common among diabetic patients [17]. T2DM is also known to contribute in LV geometric changes, commonly concentric pattern, despite of patients' hypertensive status. Hyperinsulinemia and hyperglycemia might play role on this finding [17]. Therefore diabetes was included as confounding factors in the adjusted model, but the results showed that CAD was still associated with higher LV mass and volume independent of T2DM status.

Loss of association between LV remodeling parameters and CAD after HF was included as the confounder showed that CAD association with LV remodeling was dependent on the occurrence of HF (reduced systolic function). Hypertensive patients with CAD which had developed HF had higher mass and volume. Therefore remodeling effect of CAD was fully explained by the presence of HF. As we know, CAD was the primary risk factor for HF development itself [18]. In the absence of MI, it is uncommon for concentric hypertrophy from hypertension progress to dilated cardiac failure (HF with reduced ejection fraction) [19]. Both the chronic and acute ischemia cause direct damage to the myocardium which leads to scar formation and changes in cardiac structure [19]. $\mathrm{HF}$, defined in this study as reduced ejection fraction, was also known to have a close relation with LV remodeling. In heart with worsening systolic function, LV shape usually become more dilated and hypertrophied whereas reverse of these remodeling was associated with improved cardiac function and better prognosis [20]. Pathologic LV remodeling is closely related to sympathetic activities, RAA system, oxidative stress and pro-inflammatory cytokines which are upregulated after both myocardial injury and also increase wall stress as seen on HF setting.1 Thus, insignificant association after adjustment of HF factor can be well explained, therefore HF was not included as confounder in this study, but as an explanatory model for cardiac remodeling in hypertensive patients with CAD.
Increased RWT has been reported to be associated with higher levels of epinephrine, aldosterone, and growth factors which also found increased in CAD [21], [22]. Non-significant difference between both groups might be explained by the fact that we did not exclude MI patients in the CAD groups and that the calculation of RWT itself was influenced by the infarcted wall thickness. Thus, based on the previous explanation, MI might cause uneven proportion of wall thickness as posterior wall thickness was the main element of defining RWT by echocardiography. This issue was addressed in a study by Seko et al. [21]

Cardiac remodeling due to CAD starts in the endothelium and progressively forming atherosclerotic plaque. Plaque causing ischemia and luminal narrowing leads to microvascular response and perpetuates elevated BP which finally provokes cardiac remodeling. Myocardial remodeling involves all tissue components, including myocytes, interstitial cell, such as excitationcontraction coupling, neurohormonal activation, and alteration of interstitial matrix [8]. Myocyte loss due to myocardial injury in CAD acts as initial stimulus for ventricular enlargement, rendering an area akinetic or dyskinetic. While other remaining normal myocardium overworked to compensate for the decline in cardiac function. This leads to further increase in ventricular mass, an initial adaptive way to decrease myocardial wall stress according to the law of Laplace as additional to previously hypertrophic wall due to hypertension. Enlargement of LV volume contributes toward the preservation of stroke volume which corresponds to Starling's law [23].

Cardiac remodeling consists of pathological substrate that might have clinical impact for the onset and progression of cardiac dysfunction. Although asymptomatic at first, it might evolve to sign and symptoms of HF. A review by Pimentel et al. stated that significant part of deaths associated with cardiac remodeling was caused by sudden death, even in asymptomatic patients [3]. Hence, cardiac remodeling itself should serve as target therapy for most of $\mathrm{HF}$ medications. For the purpose of early detection of LV remodeling after CAD, future study on hypertensive patients without HF is warranted. Follow-up of this population may eventually provide important prognostic information.

\section{Conclusion}

CAD in hypertensive subjects was associated with alteration of LV remodeling, such as higher LV mass and volume, independent of age, hypertensive duration, consumption of hypertension medication, and T2DM. These associations were largely explained by the occurrence of HF with reduced ejection fraction. CAD, however, was not associated with RWT. 


\section{Strength and Limitation}

To the best of our knowledge, this is among the first study in this population and the findings may serve as the foundation for future studies. This result might also represented real clinical setting situation, where the comorbidities commonly overlap one to another. We acknowledge several potential limitation of this study. First, study design was cross-sectional study, thus we only can pictured the association, not the plausible causal effect relationship of CAD and LV remodeling. Second, since CAD was diagnosed only by history taking or clinicians' written diagnosis and information based on coronary angiography was available, it is likely that the true prevalence of CAD was underestimated and the duration of CAD was uncertain. Thirdly, due to limited statistical power, we did not able to control for comorbidities objectively (which can cause bias alteration of LV remodeling, such as CKD and T2DM) besides from clinician diagnosis on medical report in the analysis. Fourth, since hypertensive status was only based on measurements at 1-time visit for patients without the history of hypertension, hypertension might be over diagnosed. Finally, incomplete data of hypertension duration in our study may hamper the analysis since it may relate to both $C A D$ and cardiac remodeling which need to be addressed appropriately.

\section{Consent to Participate}

All patients consent was obtained.

\section{Human and Animal Rights}

No animals were used in this study. All human procedures were followed in accordance with the principles of the Declaration of Helsinki.

\section{Acknowledgment}

We would like to thank all patients who participated in this study.

\section{References}

1. Konstam MA, Kramer DG, Patel AR, Maron MS, Udelson JE. Left ventricular remodeling in heart failure: Current concepts in clinical significance and assessment. JACC Cardiovasc Imaging. 2011;4(1):98-108. https://doi.org/10.1016/j.jcmg.2010.10.008 PMid:21232712

2. Anand IS, Florea VG, Solomon SD, Konstam MA, Udelson JE. Noninvasive assessment of left ventricular remodeling: Concepts, techniques, and implications for clinical trials. J Card Fail. 2002;8(Suppl):S452-64. https://doi.org/10.1054/ jcaf.2002.129286

PMid: 12555158

3. Azevedo PS, Polegato BF, Minicucci MF, Paiva SAR, Zornoff LAM. Cardiac remodeling: Concepts, clinical impact, pathophysiological mechanisms and pharmacologic treatment. Arq Bras Cardiol. 2016;106(1):62-9. https://doi.org/10.5935/ abc. 20160005

PMid:26647721

4. Haider AW, Larson MG, Benjamin EJ, Levy D. Increased left ventricular mass and hypertrophy are associated with increased risk for sudden death. J Am Coll Cardiol. 1998;32(5):1454-9. https://doi.org/10.1016/s0735-1097(98)00407-0

PMid:9809962

5. Verma A, Meris A, Skali H, Ghali JK, Arnold JM, Bourgoun M, et al. Prognostic implications of left ventricular mass and geometry following myocardial infarction: The VALIANT (VALsartan In Acute myocardial iNfarcTion) echocardiographic study. JACC Cardiovasc Imaging. 2008;1(5):582-91. https://doi. org/10.1016/j.jcmg.2008.05.012

PMid: 19356485

6. Levy D, Garrison RJ, Savage DD, Kannel WB, Castelli WP. Prognostic implications of echocardiographically determined left ventricular mass in the Framingham heart study. $\mathrm{N}$ Engl J Med. 1990;322(22):1561-6. https://doi.org/10.1056/ nejm199005313222203

PMid:2139921

7. Restini CB, Garcia AF, Natalin HM, Natalin GM, Rizzi E. Signaling Pathways of Cardiac Remodeling Related to Angiotensin II. Renin-Angiotensin System - Past, Present and Future; 2017. https://doi.org/10.5772/66076

8. Heusch G, Libby P, Gersh B, Yellon D, Böhm M, Lopaschuk G, et al. Cardiovascular remodeling in coronary artery disease and heart failure. Lancet 2014;383(9932):1933-43. https://doi. org/10.1016/s0140-6736(14)60107-0

PMid:24831770

9. Uçar H, Gür M, Börekçi A, Yıldırım A, Baykan AO, Kalkan GY, et al. Relationship between extent and complexity of coronary artery disease and different left ventricular geometric patterns in patients with coronary artery disease and hypertension. Anatol J Cardiol. 2015;15(10):789-94. https://doi.org/10.5152/ akd.2014.5747 PMid:25592099

10. Fernandes-Silva MM, Shah AM, Hedge S, Goncalves A, Claggett $\mathrm{B}$, Cheng $\mathrm{S}$, et al. Race-related differences in left ventricular structural and functional remodeling in response to increased afterload: The atherosclerosis risk in communities study. JACC Heart Fail. 2017;5(3):157-65. https://doi. org/10.1016/j.jchf.2016.10.011

PMid:28017356

11. Marwick TH, Gillebert TC, Aurigemma G, Chirinos J, Derumeaux G, Galderisi M, et al. Recommendations on the use of echocardiography in adult hypertension: A report from the European Association of Cardiovascular Imaging (EACVI) and the American Society of Echocardiography (ASE). Eur Heart J Cardiovasc Imaging. 2015;16(6):577-605. https://doi. org/10.1016/j.echo.2015.05.002 PMid:25995329

12. Zabalgoitia M, Berning J, Koren MJ, Støylen A, Nieminen MS Dahlöf $\mathrm{B}$, et al. Impact of coronary artery disease on left 
ventricular systolic function and geometry in hypertensive patients with left ventricular hypertrophy (the LIFE study). Am J Cardiol. 2001;88(6):646-50. https://doi.org/10.1016/ s0002-9149(01)01807-0

\section{PMid:11564388}

13. Kishi S, Magalhaes TA, George RT, Dewey M, Laham RJ, Niinuma $\mathrm{H}$, et al. Relationship of left ventricular mass to coronary atherosclerosis and myocardial ischaemia: The CORE320 multicenter study. Eur Heart J Cardiovasc Imaging. 2015;16(2):166-76. https://doi.org/10.1093/ehjci/jeu217 PMid:25368207

14. Drazner $\mathrm{MH}$. The progression of hypertensive heart disease. Circulation. 2011;123(3):327-34.

15. Heidland UE, Strauer BE. Left ventricular muscle mass and elevated heart rate are associated with coronary plaque disruption. Circulation. 2001;104(13):1477-82. https://doi. org/10.1161/hc3801.096325

\section{PMid:11571239}

16. Pierdomenico SD. Left-ventricular hypertrophy and coronary artery disease. Am J Hypertens. 2007;20(10):1036-7. https:// doi.org/10.1016/j.amjhyper.2007.06.002

\section{PMid:17903684}

PMid:21232712

17. Maiello M, Zito A, Carbonara S, Ciccone MM, Palmiero P. Left ventricular mass, geometry and function in diabetic patients affected by coronary artery disease. J Diabetes Complications. 2017;31(10):1533-7. https://doi.org/10.1016/j. jdiacomp.2017.06.014

PMid:28890308
18. Lala A, Desai AS. The role of coronary artery disease in heart failure. Heart Fail Clin. 2014;10(2):353-65. https://doi. org/10.1016/j.hfc.2013.10.002

\section{PMid:24656111}

19. Hajouli S, Ludhwani D. Heart Failure And Ejection Fraction. Treasure Island, FL: StatPearls Publishing; 2021.

20. Aimo A, Gaggin HK, Barison A, Emdin M, Januzzi JL. Imaging biomarker, and clinicalpredictors of cardiac remodelingin heart failure with reduced ejection fraction. JACC Heart Fail. 2019;7(9):782-94. https://doi.org/10.1016/j.jchf.2019.06.004 PMid:31401101

21. Seko $Y$, Kato T, Morita $Y$, Yamaji $Y$, Haruna $Y$, Izumi T, et al. Impact of left ventricular concentricity on long-term mortality in a hospitalbased population in Japan. PLoS One. 2018;13(8):e0203227. https://doi.org/10.1371/journal.pone.0203227 PMid:30161243

22. Bang CN, Gerdts E, Aurigemma GP, Boman K, de Simone G, Dahlöf $\mathrm{B}$, et al. Four-group classification of left ventricular hypertrophy based on ventricular concentricity and dilatation identifies a low-risk subset of eccentric hypertrophy in hypertensive patients. Circ Cardiovasc Imaging. 2014;7(3):4229. https://doi.org/10.1161/CIRCIMAGING.113.001275

PMid:24723582

PMid:24831770

23. Cokkinos DV, Belogianneas C. Left ventricular remodeling: A problem in search of solutions. Eur Cardiol. 2016;11(1):29-35 https://doi.org/10.15420/ecr.2015:9:3

PMid:30310445 